University of Nebraska - Lincoln

DigitalCommons@University of Nebraska - Lincoln

Faculty Publications from Nebraska Center for Materials and Nanoscience

Materials and Nanoscience, Nebraska Center for (NCMN)

February 2004

\title{
Ultrasound diffusion for crack depth determination in concrete
}

S.K. Ramamoorthy

University of Nebraska - Lincoln

Y. Kane

University of Nebraska - Lincoln

Joseph A. Turner

University of Nebraska - Lincoln, jaturner@unl.edu

Follow this and additional works at: https://digitalcommons.unl.edu/cmrafacpub

Part of the Nanoscience and Nanotechnology Commons

Ramamoorthy, S.K.; Kane, Y. ; and Turner, Joseph A., "Ultrasound diffusion for crack depth determination in concrete" (2004). Faculty Publications from Nebraska Center for Materials and Nanoscience. 59. https://digitalcommons.unl.edu/cmrafacpub/59

This Article is brought to you for free and open access by the Materials and Nanoscience, Nebraska Center for (NCMN) at DigitalCommons@University of Nebraska - Lincoln. It has been accepted for inclusion in Faculty Publications from Nebraska Center for Materials and Nanoscience by an authorized administrator of DigitalCommons@University of Nebraska - Lincoln. 


\title{
Ultrasound diffusion for crack depth determination in concrete
}

\author{
S. K. Ramamoorthy, Y. Kane, and J. A. Turner ${ }^{\mathrm{a})}$ \\ Department of Engineering Mechanics, W317.4 Nebraska Hall, University of Nebraska-Lincoln, \\ Lincoln, Nebraska 68588-0526
}

(Received 2 July 2003; revised 15 November 2003; accepted 24 November 2003)

\begin{abstract}
The determination of the depth of surface-breaking cracks in concrete specimens using an ultrasound diffusion technique is discussed. Experiments were carried out on precracked concrete specimens of varying crack depths $(0 \%-40 \%$ of the specimen thickness). Contact transducers were placed at the specimen surface with source and receiver separated by the crack. Tone-burst excitations over a frequency range of $400-600 \mathrm{kHz}$ were used. At these frequencies, ultrasound is scattered considerably by the heterogeneities in the concrete. In the limit of many scattering events, the evolution of energy may be modeled as a diffusion process. The arrival of the peak diffuse energy at the receiver is delayed due to the presence of crack. This delay is the prime indicator used for determining crack depth. Numerical and analytical analyses were also used for comparison. These results are in basic agreement with the experiments. In addition, these analyses are used to study the limits of this technique. In particular, it is shown that this technique is applicable to cracks greater than the scattering mean-free path, which is estimated at about $1 \mathrm{~cm}$ for these specimens. Aspects of practical implementation are also discussed. (c) 2004 Acoustical Society of America.
\end{abstract} [DOI: $10.1121 / 1.1642625]$

PACS numbers: $43.20 . \mathrm{Gp}, 43.35 . \mathrm{Cg}, 43.35 . \mathrm{Zc}[\mathrm{SFW}]$

Pages: 523-529

\section{INTRODUCTION}

Concrete structures are continually subject to effects that degrade their structural integrity. Near-surface damage in concrete may take the form of distributed microcracks or distinct, large cracks that extend to the surface of the structure. Damage from microcracking develops at length scales that are smaller than the size of the majority of the aggregate. ${ }^{1}$ However, large cracks can result from mechanical overloading, freeze-thaw cycling, by drying shrinkage, or from the coalescence of many microcracks. Several recent research articles report efforts to determine nondestructively the depth of surface-breaking cracks in concrete using elastic stress wave time-of-flight (wave velocity) techniques. ${ }^{2-4}$ In these papers, the crack depth is determined if the velocity of wave propagation in the intact concrete is known and a particular wave path and wave pulse-crack interaction are assumed. The frequency range for these experiments varied from 0 to $100 \mathrm{kHz}$. A frequency of $100 \mathrm{kHz}$ corresponds to a wavelength on the order of a few centimeters in concrete. In the impact-echo method, ${ }^{2}$ the user must interpret the recorded echo signals. Quality interpretation requires a user experienced in waveform recognition. Other researchers use frequencies in the range of 0 to $70 \mathrm{kHz}{ }^{5,6}$ The wave source is due to the impact of a steel ball on the specimen surface, as in the impact-echo method. Surface wave methods have also been used to characterize the microstructural properties of concrete such as grain size, porosity, or microcrack distribution. ${ }^{7}$ The penetration depth of the surface waves is on the order of one wavelength. Hence, methods that utilize surface waves are, in general, insensitive to deep cracks. To detect deep cracks by surface wave methods, low-frequency

\footnotetext{
${ }^{\text {a) }}$ Author to whom correspondence should be addressed. Electronic mail: jaturner@unl.edu
}

waves must be used. These can be difficult to generate with sufficient energy without damaging the surface. Ultrasonic guided waves (Lamb waves) have also been used to detect damage in concrete beams and to detect delaminations between steel bars and concrete. ${ }^{8}$ However, guided wave testing is complicated by the presence of the many available modes and by the difficulties associated with deep slabs. It is also difficult to generate a low-frequency guided wave using commercial transducers.

The response of a heterogeneous elastic body to a propagating wave is a function of the wavelength in comparison with the length scale of the heterogeneities. When wavelengths on the order of the microstructure length scale are used, a large amount of scattering occurs. The scattering process causes the elastic wave energy to propagate in directions which do not coincide with the incident wave. The propagating wave is attenuated due to these scattering losses. The scattering process is generally energy conserving - the scattered energy is not lost, but is no longer in phase with the exciting wave. Additional attenuation is caused by true dissipative mechanisms. Thus, the material response becomes a combination of coherent and diffuse (incoherent) energy. If the initial energy scatters many times during its path from source to receiver, it is expected to behave as a diffusion process. Previous one-dimensional diffusion experiments in concrete have shown that the ultrasonic diffusivity and dissipation may be extracted from such measurements. ${ }^{9}$ In many cases, the evolution of the diffuse energy occurs on a much longer time scale than the time necessary for the coherent wave to propagate across the specimen.

In this article, a new technique is discussed to determine the depth of surface cracks in concrete by exploiting the multiple scattering properties of the concrete. In the next section, the concrete specimens are discussed. In Sec. III, the 


\begin{tabular}{cccc}
\hline \hline Specimen ID & Dimensions $(\mathrm{cm})$ & Crack depth $(\mathrm{cm})$ & $\begin{array}{c}\text { \% Crack depth } \\
\text { to slab depth }\end{array}$ \\
\hline Slab 1 & $60.96 \times 60.96 \times 20.32$ & 0 & 0 \\
Slab 2 & $60.96 \times 60.96 \times 20.32$ & 1.27 & 6.25 \\
Slab 3 & $60.96 \times 60.96 \times 20.32$ & 3.81 & 18.75 \\
Slab 4 & $60.96 \times 60.96 \times 20.32$ & 7.62 & 37.50 \\
\hline \hline
\end{tabular}

results for the uncracked slab are presented. The onedimensional diffusion results ${ }^{9}$ are extended to two dimensions representative of the slab geometry considered here. The ultrasonic diffusivity and dissipation were determined from experiments by comparing the experimental results with an analytical solution of the two-dimensional diffusion process. These parameters may also be extracted from experiments on cracked slabs if a location away from the major crack is used. For all measurements, the diffuse energy field was determined by low-pass filtering the square of the transducer response. In addition, these results were compared with the solution obtained numerically from the twodimensional diffusion equation. In Sec. IV, the analysis for the slabs with a major crack is presented. The experiments were conducted on three cracked specimens. The time shift of the peak amplitude of the diffuse energy can be used to determine the depth of the surface-breaking crack. Based on dimensional analysis, the time shift is expected to be proportional to the square of the crack depth divided by the diffusivity. This scaling with crack depth has been confirmed. The experimental values were in agreement with the numerical results. The potential advantages of this technique over other methods include the ease of excitation at high frequencies and the sensitivity to deep cracks. Also, it is not necessary to determine the wave velocity and other wave propagation parameters (wave path, interaction with geometry of the slab). The limits of this technique are its insensitivity to shallow cracks and possibly low signal-to-noise ratio, although sufficient repetition averaging can be used to overcome the latter.

\section{SPECIMEN PREPARATION}

Four specimens were cast with $47 \mathrm{BD}$ bridge deck slab concrete having a compressive strength of $35 \mathrm{MPa}$ with varying notch depth $(0 \%-40 \%)$ of the thickness. The notches were created by placing a steel plate of 3-mm thickness in the mold before casting it with concrete. Cast oil was applied to both sides of the steel plate to facilitate easy removal. After the initial setting time, the steel plates were removed from the mold and the specimens were covered with burlap. After $24 \mathrm{~h}$, the specimens were demolded and cured in a fog room for the next 28 days in order to reduce the likelihood of shrinkage cracks. Specific details of the specimens are shown in Table I.

\section{UNCRACKED SLABS}

Previously, the diffusion of ultrasound in concrete cylinder specimens was modeled as a one-dimensional process which does not apply to the slab geometry of interest here. ${ }^{9}$ To recover the diffusivity and dissipation, the diffuse energy evolution was modeled as a two-dimensional diffusion process. The analytical solution was compared with the experimental solution to determine the scattering properties of the concrete. In this section, the extension of the onedimensional results to the two-dimensional diffusion problem relevant for these slabs is discussed in terms of theory, numerical modeling, and experiments.

\section{A. Analytical solution}

The diffusion of ultrasonic energy through a concrete slab with no crack is modeled by the two-dimensional diffusion equation with dissipation. The equation is given by

$$
\begin{aligned}
& D\left(\frac{\partial^{2}}{\partial x^{2}}+\frac{\partial^{2}}{\partial y^{2}}\right)\langle E(x, y, t)\rangle-\frac{\partial}{\partial t}\langle E(x, y, t)\rangle-\sigma\langle E(x, y, t)\rangle \\
& \quad=f(x, y, t),
\end{aligned}
$$

with forcing condition

$$
f(x, y, t)=E_{0} \delta\left(x-x_{0}\right) \delta\left(y-y_{0}\right) \delta\left(t-t_{0}\right),
$$

where $E(x, y, t)$ is the ultrasonic spectral density (energy per area, per frequency bandwidth), $D$ is the ultrasonic diffusivity with dimension length squared per time, $\sigma$ is the dissipation with dimension inverse time, and $E_{0}$ is the initial energy which is deposited at time $t=0$. Equation (1) describes the evolution of the ensemble average energy density for an infinite medium. For the case considered here, Neumann boundary conditions (zero flux across the boundaries) are enforced at the specimen boundaries. The series solution of Eq. (1) for the source located at $x_{0}$ and $y_{0}$ is given by

$$
\begin{aligned}
E(x, y, t)= & \left\{1+\sum_{n=1}^{\infty} \sum_{m=1}^{\infty} 4 \cos \left(\frac{n \pi x_{0}}{l}\right) \cos \left(\frac{m \pi y_{0}}{p}\right)\right. \\
& \times \cos \left(\frac{n \pi x}{l}\right) \cos \left(\frac{m \pi y}{p}\right) e^{-D\left[(n \pi / l)^{2}+(m \pi / p)^{2}\right] t} \\
& +\sum_{n=1}^{\infty} 2 \cos \left(\frac{n \pi x_{0}}{l}\right) \cos \left(\frac{n \pi x}{l}\right) e^{-D\left[(n \pi / l)^{2}\right] t} \\
& \left.+\sum_{m=1}^{\infty} 2 \cos \left(\frac{m \pi y_{0}}{p}\right) \cos \left(\frac{m \pi y}{p}\right) e^{-D\left[(m \pi / p)^{2}\right] t}\right\} \\
& \times E_{0} e^{-\sigma t},
\end{aligned}
$$

where $l$ and $p$ are the lateral dimensions of the slab.

Figure 1 shows example energy curves calculated using Eq. (3) for different combinations of ultrasonic diffusivity and dissipation values using $l=0.6 \mathrm{~m}$ and $p=0.2 \mathrm{~m}$. The vertical positions of the source and receiver are identical $(y$ $=y_{0}=0.2 \mathrm{~m}$ ), while the horizontal positions are equidistant from the center of the slab and separated by $6 \mathrm{~cm}\left(x_{0}\right.$ $=0.27 \mathrm{~m}$ and $x=0.33 \mathrm{~m}$ ) Steeper curves correspond to higher dissipation. The peak arrival time of the diffuse energy is an important parameter in determining the depth of the surface-breaking crack. Figure 1 shows that this peak arrival time decreases for higher values of dissipation. 


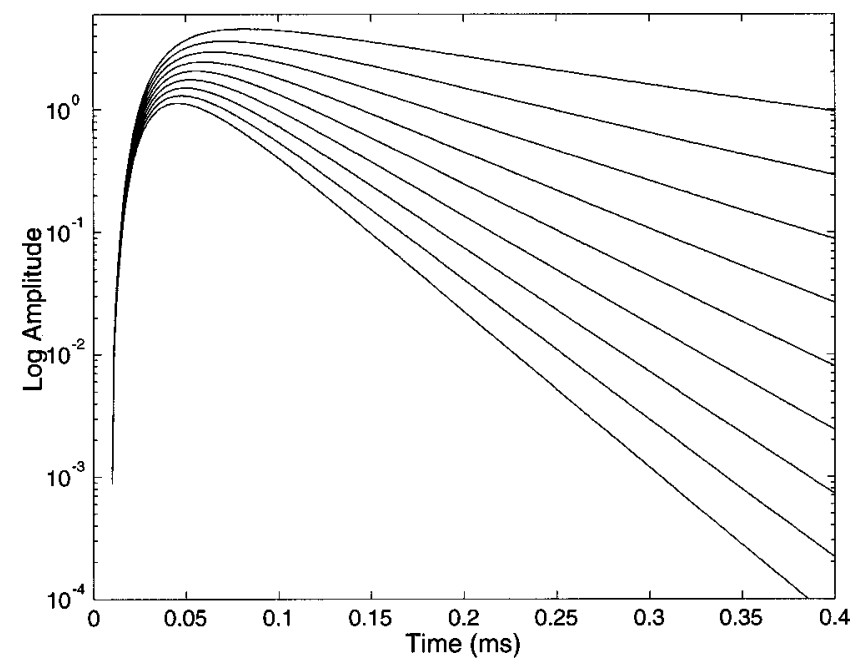

FIG. 1. Analytical solution, Eq. (3), for diffusivity of $12 \mathrm{~m}^{2} / \mathrm{s}$ and dissipation varying from 3000 to $270001 / \mathrm{s}$ assuming slab dimensions $l=0.6 \mathrm{~m}$ and $p=0.2 \mathrm{~m}$. The vertical positions of the source and receiver are identical $\left(y=y_{0}=0.2 \mathrm{~m}\right)$, while the horizontal positions are equidistant from the center of the slab and separated by $6 \mathrm{~cm}\left(x_{0}=0.27 \mathrm{~m}\right.$ and $\left.x=0.33 \mathrm{~m}\right)$.

\section{B. Numerical solution}

A finite-element code was developed for solution of the diffusion equation given by Eq. (1) with forcing condition given by Eq. (2). The cross-sectional details of the experimental specimens given in Table I were used for creating a rectangular domain for this model. The rectangular domain was discretized and meshed with bilinear rectangular elements, and the boundary of the domain was modeled with the appropriate Neumann boundary conditions. By using the divergence theorem, the weak formulation of the diffusion Eq. (1) is given by

$\int\left[\nu\left(\frac{\partial E}{\partial t}-\sigma E-E_{0}\right)+D \frac{\partial \nu}{\partial x} \frac{\partial E}{\partial x}+D \frac{\partial \nu}{\partial y} \frac{\partial E}{\partial y}\right] d x d y=0$,

where $\nu$ is the weight function, chosen from the basis function $\psi_{j}^{e}(x, y)$ used for calculating the approximate energy, $E$, and $E_{0}$ is the initial energy deposited. The finite-element approximation is given by

$$
E(x, y, t) \approx \sum_{j=1}^{n} E_{j}^{e}(t) \psi_{j}^{e}(x, y),
$$

where $E_{j}$ is the value of $E(x, y, t)$ at a point $\left(x_{j}, y_{j}\right)$ at time $t$ and $\psi_{j}^{e}(x, y)$ is the basis function. In matrix form, the transient energy equation at the elemental level is given by

$$
\left[M^{e}\right]\left\{E^{e}\right\}+\left[K^{e}\right]\left\{E^{e}\right\}=\left\{f^{e}\right\} .
$$

A forward difference scheme was used to solve Eq. (6). The time step $\Delta t$ for the numerical analysis was chosen such that the stability criterion given by

$$
\Delta t<\Delta t_{c r}=\frac{2}{\lambda_{\max }},
$$

is satisfied. In Eq. (7), $\lambda_{\max }$ is the largest eigenvalue of $\left[M^{-1}\right][K]$, where $[M]$ and $[K]$ are the global mass and stiffness matrices, respectively.

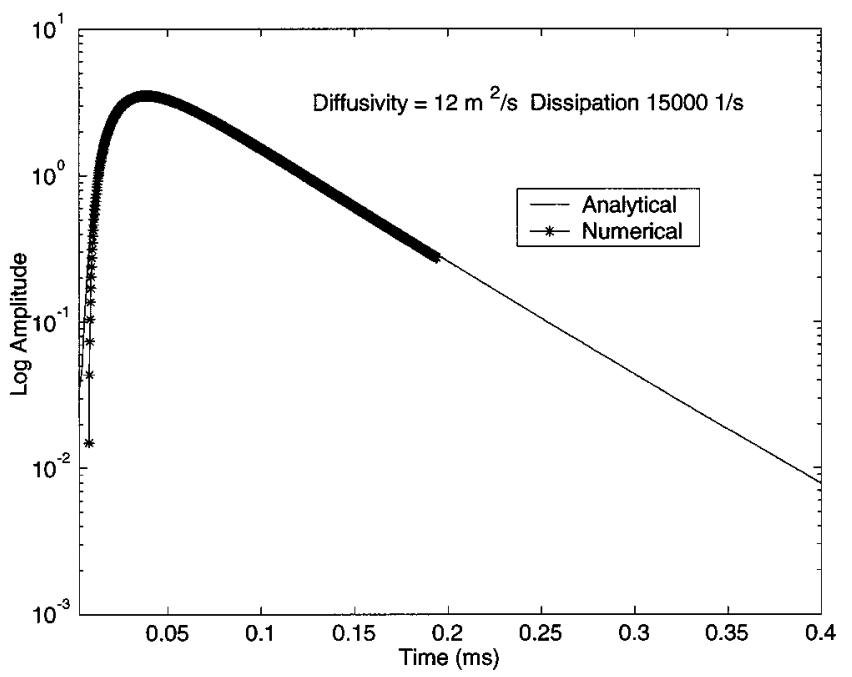

FIG. 2. Diffuse energy for slab 1 (uncracked) by analytical and numerical methods for a separation distance of $6 \mathrm{~cm}$.

The typical time step used in the numerical analysis ranged from 0.2 to $2 \mu \mathrm{s}$. To check for convergence of the code, the analysis was carried out by varying the number of elements in the rectangular domain. It was found that the numerical solution compared well with the analytical solution (with a relative error of a few microseconds for the peak arrival time) for 1200 elements. Increasing the number of elements to 2400 increased the computation time considerably and did not significantly affect the peak arrival time. Since the main measure of crack depth is the peak arrival time, 1200 elements was judged sufficient for resolving this peak time. Figure 2 shows the comparison of the diffuse energy obtained by analytical and numerical methods for $D$ $=12 \mathrm{~m}^{2} / \mathrm{s}$ and $\sigma=150001 / \mathrm{s}$. The peak arrival time depends on the diffusion parameters $D$ and $\sigma$ as well as the sourcereceiver separation distance. This dependence is illustrated in Fig. 3. The peak arrival time, calculated numerically, is plotted versus source-receiver separation distance for various combinations of $D$ and $\sigma$. The peak arrival time varies from tens of $\mu$ s to hundreds of $\mu$ s. It can also be seen that the

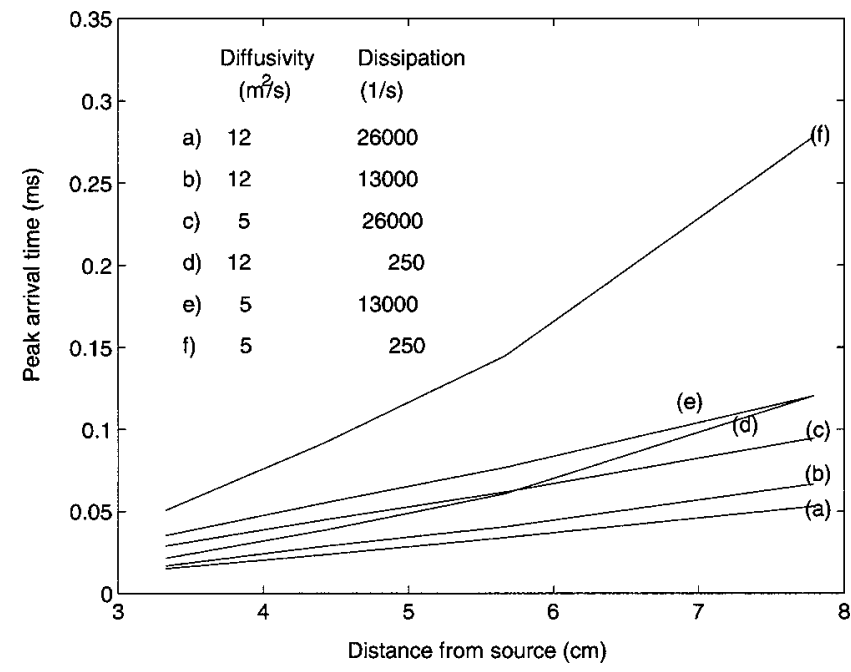

FIG. 3. Numerical results for peak arrival time as a function of sourcereceiver separation and ultrasonic diffusion parameters $D$ and $\sigma$. 


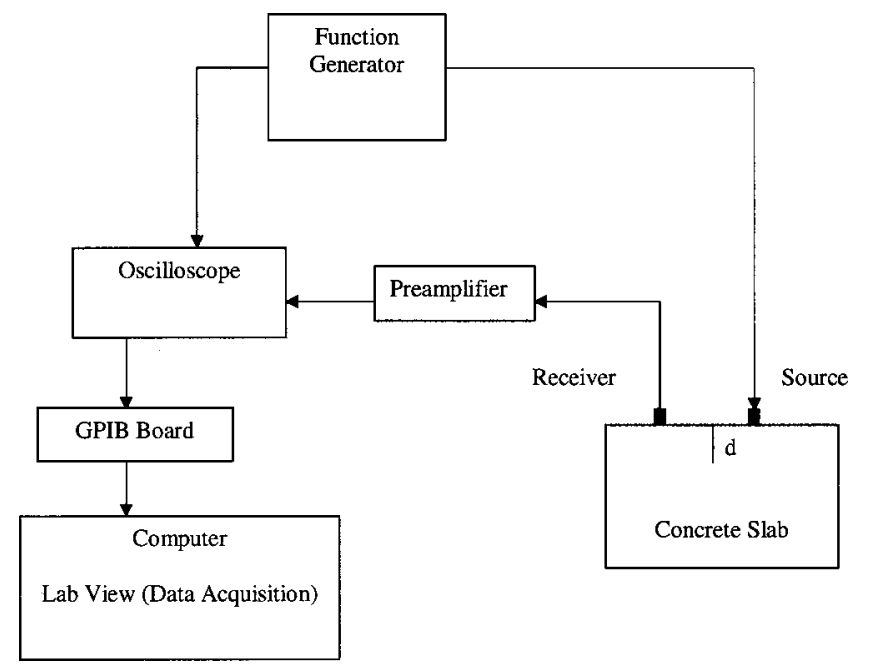

FIG. 4. Schematic layout of experimental setup.

separation distance is more critical, in terms of peak arrival time, for certain combinations of $D$ and $\sigma$.

\section{Experiments}

Experiments were first conducted on slab 1 (without a crack) for comparison with the analytical solution given by Eq. (1). The schematic layout of the ultrasound diffusion experiment is shown in Fig. 4. The source transducer, a 25.4-mm Panametrics contact transducer with a central frequency of $500 \mathrm{kHz}$, was coupled to the top surface of the slab. The receiving transducer, a Valpey-Fisher point-like pin transducer $(2.5-\mathrm{mm}$ diameter) was located at a separation distance $r$ from the center of the source. Both the source and receiver were coupled to the specimen with a viscous couplant. The diffuse energy was obtained for different separation distances. The transducers were placed away from the edges to avoid edge effects. The source transducer was excited with a 5-cycle tone burst over a frequency range of $400-600 \mathrm{kHz}$ using an arbitrary waveform generator. The output from the receiving transducer was preamplified and then digitized by an oscilloscope. To improve the signal-tonoise ratio, repetition averaging was used (500 averages). The oscilloscope was connected to a personal computer through a GPIB board and a LABVIEW program was used to acquire the average waveform. The square of the waveform data was then filtered using a low-pass filter. The result is a quantity proportional to the diffuse energy. The diffusivity and dissipation of the concrete slab were obtained by comparing the energy data with the analytical and numerical results.

Figure 5 shows the comparison of the experimental diffuse energy with the analytical and numerical results for an input frequency of $500 \mathrm{kHz}$ at a source-receiver separation distance of $6 \mathrm{~cm}$. The experimental results behave according to the proposed model only up until about $0.2 \mathrm{~ms}$. After that time, the decay is no longer exponential. This type of behavior was often observed and is presumed due to the variation of dissipation within the frequency band. At these frequencies, the change of dissipation can be dramatic. ${ }^{9}$ The analytical results were computed using 50 terms in Eq. (3). No

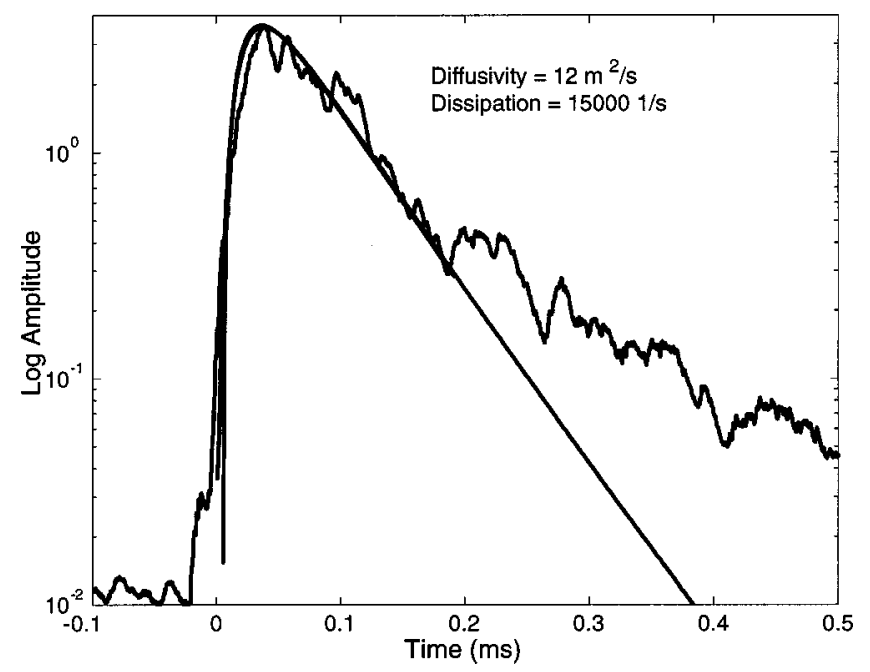

FIG. 5. Comparison of experimental data with analytical and numerical results to recover the scattering coefficients.

significant change occurs in the solution if the number of terms is increased beyond 50 . The diffusivity and the dissipation in Eq. (3) were adjusted until the analytical and experimental curves were best matched. Since the initial energy deposited, $E_{0}$, is not known, the amplitude of the analytical solution was scaled to match with the experimental data. The scattering parameters obtained by comparing the experimental data with the analytical solution for different separation distances were in agreement with the data obtained at the minimum separation distance between the source and receiver. Experiments were also carried out for the other slabs (slab 2, slab 3, and slab 4) at a location away from the surface-breaking crack. A comparison of the diffuse energy data for the different slabs confirms the assumption that the scattering properties of the concrete slab can be determined by measuring the diffuse energy field at locations away from major cracks and boundaries.

\section{CRACKED SLABS}

The cracked slabs have surface breaking cracks of varying depth (slab 2, slab 3, and slab 4). The scattering properties recovered from the uncracked slab were used to determine the predicted peak arrival time in the numerical analysis for different crack depths. The lag time, defined as the difference between the peak arrival time of the diffuse energy in the cracked and uncracked regions, is the measure used here to infer the crack depth. Comparison between experimental and numerical lag times was used to evaluate this technique.

\section{A. Analytical solution}

For the cracked slab case, the governing equation remains the same. However, in addition to the Neumann boundary conditions at the slab boundaries, an additional boundary condition is imposed, i.e., no flux across the crack. Because an analytical solution for the problem of a slab with a crack is not yet available, the numerical solution was the primary measure for comparison with the experiments on cracked slabs. 

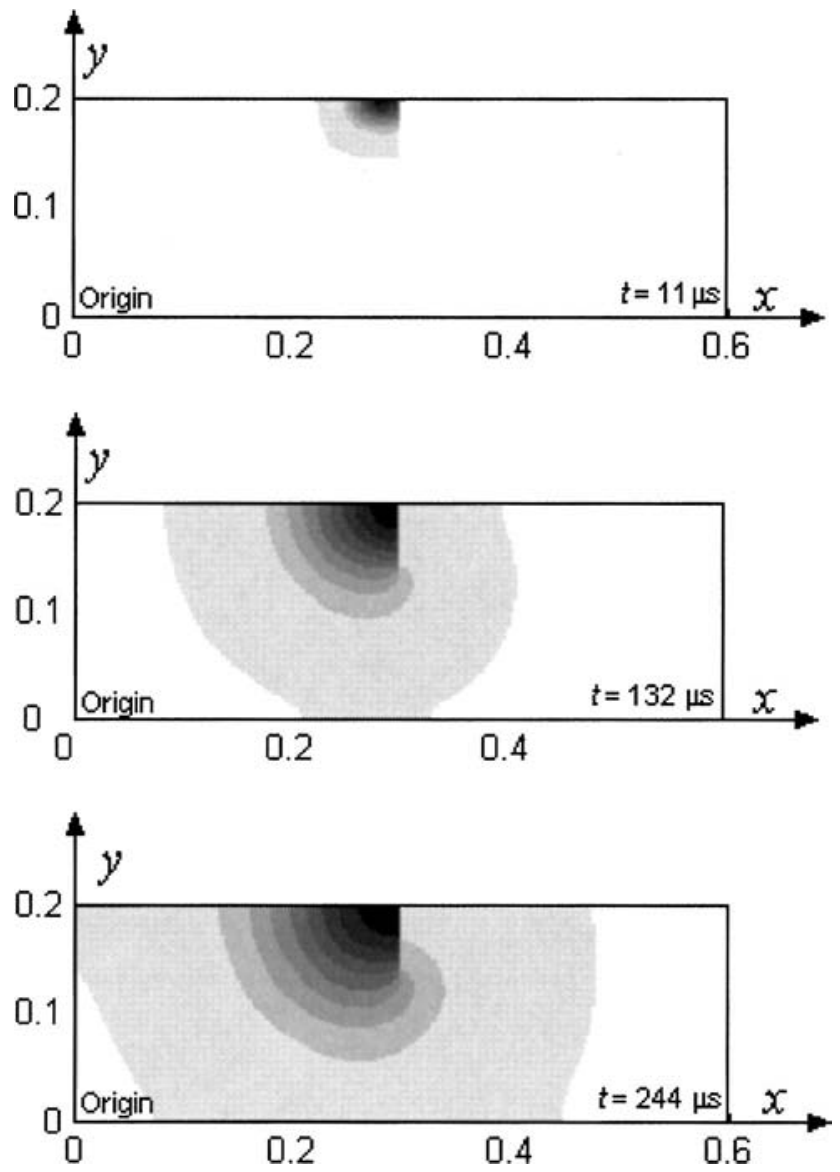

FIG. 6. Diffuse energy field for a crack depth of $30 \%$ at 11, 132, and $244 \mu$ s $\left(D=12 \mathrm{~m}^{2} / \mathrm{s}, \sigma=150001 / \mathrm{s}\right)$.

\section{B. Numerical solution}

For the cracked slabs the mesh discretization, element type, and number of elements were similar to those used for the uncracked slab. The crack was created at a given location by adding another set of nodes at the same location as existing nodes to the required crack depth. The element connectivity of the nodes at the crack location was changed such that the zero-flux condition across the crack is satisfied. Figure 6 shows the full diffuse energy field at times 11,132, and $244 \mu$ s, respectively, for a crack depth of $30 \%$ of the slab depth $\left(D=12 \mathrm{~m}^{2} / \mathrm{s}, \sigma=15000 \mathrm{1} / \mathrm{s}\right)$. The diffusion process and the delay in the arrival of the diffuse energy across the crack is clearly depicted. The analysis was carried out for different combinations of diffusivity and dissipation. The results clearly show the delay in the peak arrival time of the diffuse energy across the crack when compared with the diffuse energy for the same source-receiver separation distance at the uncracked region. Figure 7 shows the lag time for different crack depths and for different source-receiver separation distances for select values of diffusivity and dissipation. The trend is similar for all separation distances, suggesting that this distance is not critical for practical implementation. It is also clear that the lag time is a function of separation distance between the source and transducer as expected.

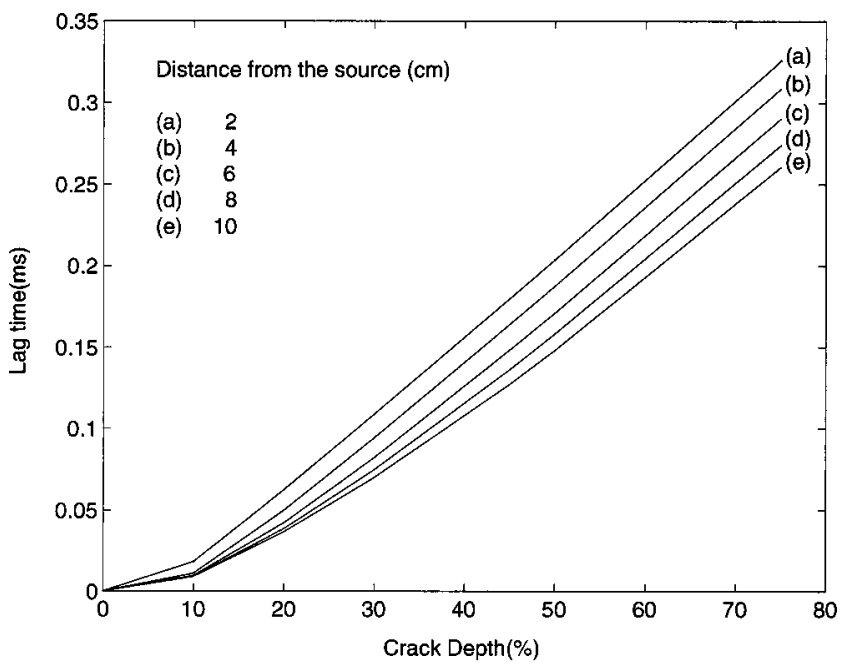

FIG. 7. Numerical results of lag time vs crack depth for different sourcereceiver separation distances $\left(D=12 \mathrm{~m}^{2} / \mathrm{s}\right.$ and $\left.\sigma=150001 / \mathrm{s}\right)$.

\section{Experiments}

The experiments on the cracked slabs were essentially identical to those for the uncracked slab. The source transducer was placed on one side of the crack and the receiver was placed on the other side of the crack as shown in Fig. 4. Figure 8 shows the waveform data obtained by the receiver for which the source and receiver were placed in an uncracked region. Figure 9 shows the waveform data for the source-receiver pair placed across the crack. Comparison of Figs. 8 and 9 shows the delay in the diffusion process clearly. There is a delay in the arrival of the peak diffuse energy when compared with the arrival of the diffuse energy obtained by receiver placed at the uncracked region of the slab. The delay in diffusion is due to the absence of flux transfer across the crack. This delay in arrival of the energy is the prime factor in assessing the depth of the surface opening crack.

The diffuse energy was obtained from the waveform data by squaring the transducer signal and filtering with a low-pass filter. The cutoff frequency for the low-pass filter

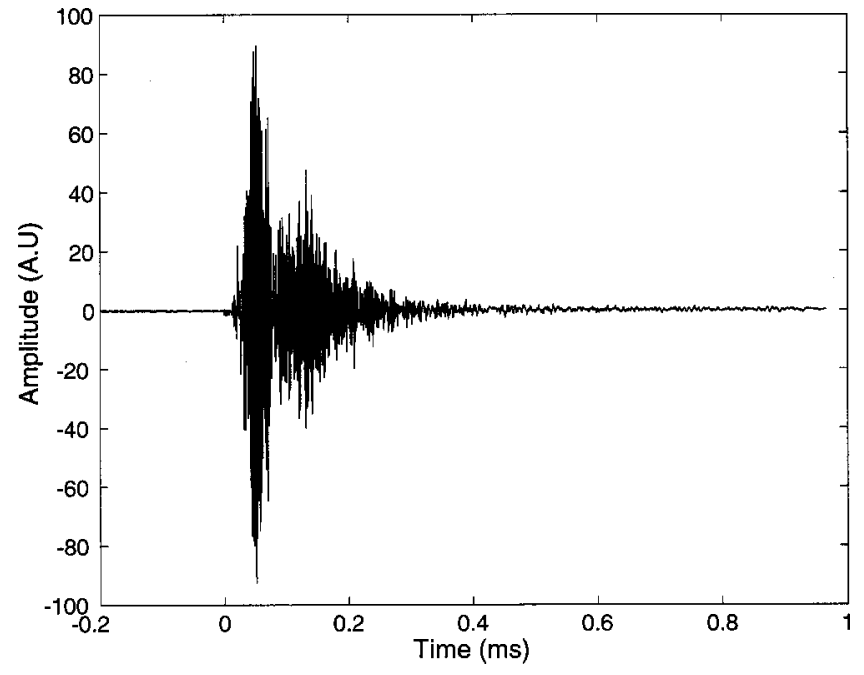

FIG. 8. Typical waveform data in an uncracked zone for an input frequency of $500 \mathrm{kHz}$ and a source-receiver separation distance of $6 \mathrm{~cm}$. 


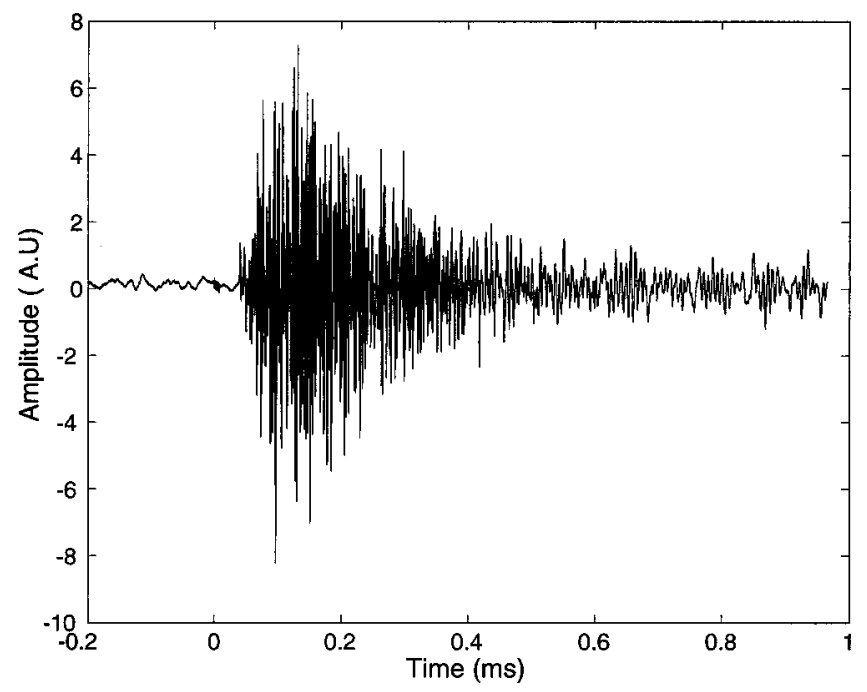

FIG. 9. Typical waveform data across the crack for an input frequency of $500 \mathrm{kHz}$ and a source-receiver separation distance of $6 \mathrm{~cm}$.

was selected by considering the stability of the energy data with respect to cutoff frequency and by considering the fluctuations in the energy data. The results were scaled such that the initial rise of the experimental and numerical data matched. Figures 10 and 11 show the comparison of the experimental diffuse energy with the numerical data for slabs 3 and 4, respectively, with a separation distance of $6 \mathrm{~cm}$ between the source and the receiver for a cutoff frequency of $10 \mathrm{kHz}$. By comparing the peak arrival time of the waveform across the crack with the peak arrival time of the data obtained at the uncracked region, the lag time for different crack depths was determined. This lag time is due to the presence of the crack. Figure 12 shows the comparison of lag time obtained by numerical analysis and by experiments for different crack depths. The error bars for the experimental lag time were determined by low-pass filtering the waveform data for three different cutoff frequencies (4, 10, and 25 $\mathrm{kHz}$ ). The standard deviation and average values of the three peak arrival times were used to calculate the error bars. For deeper cracks, the peak arrival time was more sensitive to the

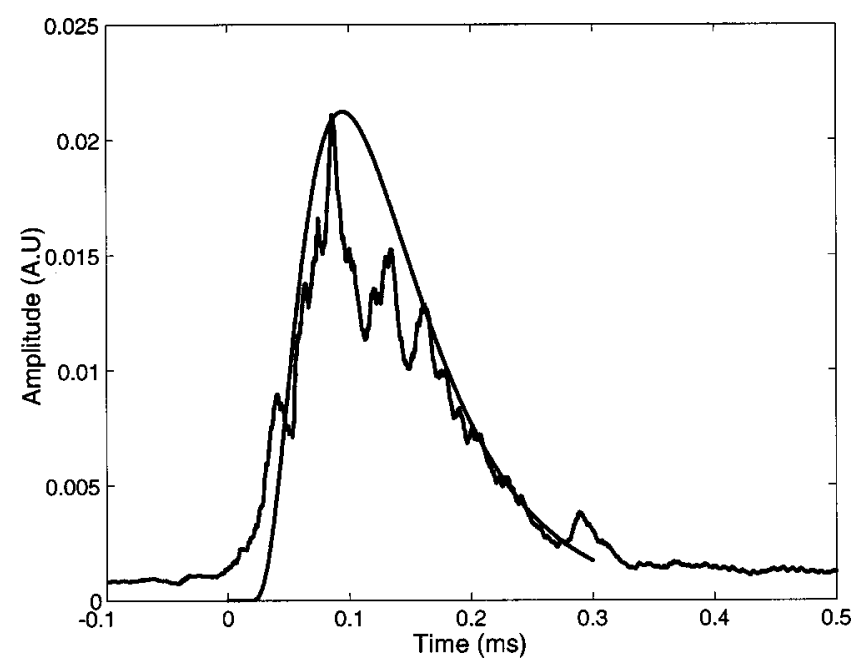

FIG. 10. Comparison of experimental diffuse energy data with numerical result for slab 3 ( $18.75 \%$ crack depth).

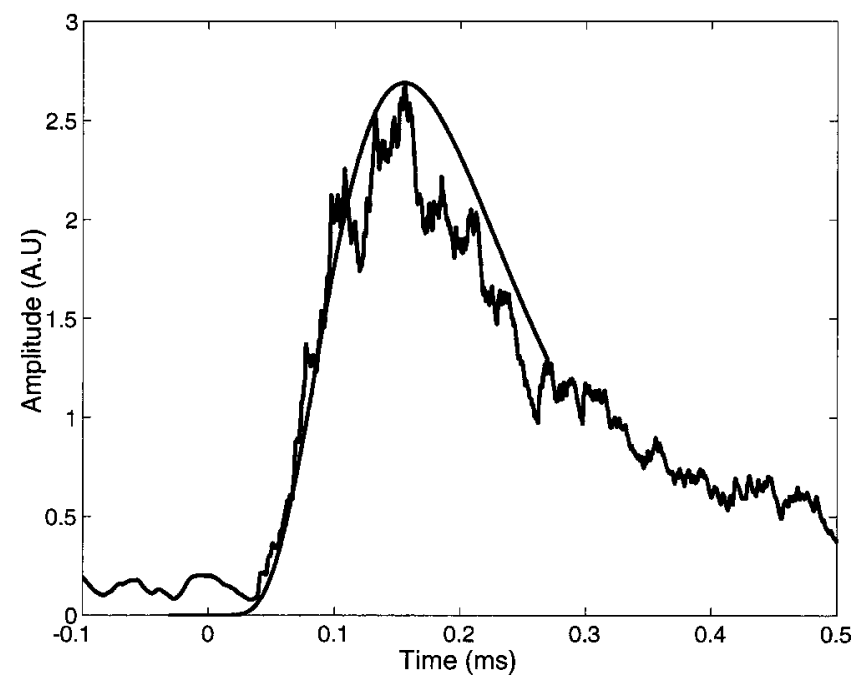

FIG. 11. Comparison of experimental diffuse energy with numerical results for slab 4 (37.5\% crack depth).

cutoff frequency than it was for shallow cracks. The agreement between the experimental results and the numerical results is very good. The inversion of experimental results to determine crack depth can occur if the diffusivity and dissipation are determined in a sample region away from boundaries and large cracks.

Trends observed in numerous numerical simulations show that the lag time can always be fit by a second-order polynomial function of crack depth. ${ }^{10}$ The quadratic term is inversely proportional to diffusivity as expected, and the linear term is inversely proportional to the dissipation. This curve must pass through the origin since there is no lag time for a zero-depth crack. Thus, there is not a constant term in this polynomial. The experimental data show that this predicted dependence does indeed hold, at least for the values of $D$ and $\sigma$ relevant for the concrete samples examined here.

\section{CONCLUSIONS}

In this article, it has been shown that in light of many scattering events the evolution of ultrasonic energy in a con-

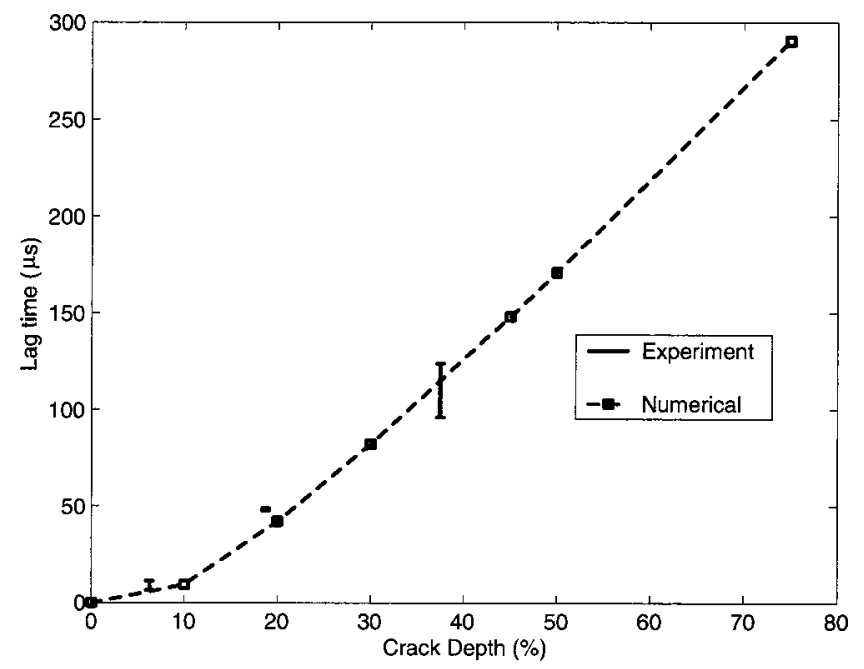

FIG. 12. Lag time vs crack depth for a source-receiver separation distance of $6 \mathrm{~cm}$. The solid line is a quadratic curve fit to the numerical data and the error bars correspond to the experimental results. 
crete slab can be modeled as a two-dimensional diffusion process. The ultrasonic diffusivity and dissipation were determined by comparing the experimental results with the analytical solution for a concrete slab without a crack. The results also compared well with the experimental results obtained in regions of the specimens that are not near boundaries or large cracks. The lag time was obtained by comparing the peak arrival time of the diffuse energy obtained at two locations, one in an uncracked region and the other across the crack. The experimental and numerical results correlated well for deeper cracks. The lag time increases considerably with increase in crack depth. The depth of the surface-breaking crack in a given concrete slab can be determined in two steps. In the first step, the scattering properties are determined from measurements in the uncracked region of the sample. In the second step, using the recovered scattering properties, the relation between the lag time and crack depth is obtained by numerical analysis. Once the relation is established, the depth of the surface-breaking crack is determined by using the experimental lag time. It should be noted that the results presented here are for notches, for which the two surfaces were not in contact. Results for real cracks with surfaces in contact are the focus of future research. The potential for characterizing nonstraight cracks and fluid-filled cracks is also under investigation.

\section{ACKNOWLEDGMENTS}

The support of the National Science Foundation (Grant Nos. CMS-9978707 and INT-0089548) and the National
Bridge Research Organization (NaBRO) at the University of Nebraska-Lincoln is gratefully acknowledged by the authors.

${ }^{1}$ S. Popovics and J. S. Popovics, "Ultrasonic testing to determine watercement ratio for freshly mixed concrete," Am. Stand. Test. Mater. 20, 262-268 (1998).

${ }^{2}$ M. Sansalone, J. M. Lin, and W. B. Streett, "Determining the depth of surface-opening cracks using impact-generated stress waves and time-offlight techniques," ACI Mater. J. 95, 168-177 (1998).

${ }^{3}$ Z. P. Bažant and J. Planas, Fracture and Size Effect in Concrete and Other Quasibrittle Materials (CRC Press, Boca Raton, 1998).

${ }^{4}$ G. Hevin, O. Abraham, H. Pedersen, and M. Campillo, "Characterisation of surface cracks with Rayleigh waves: A numerical model," NDT \& E Int. 31, 289-297 (1998).

${ }^{5}$ J. S. Popovics, W. J. Song, M. Ghandehari, K. V. Subramaniam, J. D. Achenbach, and S. P. Shah, "Application of surface wave transmission measurements for crack depth determination in concrete," ACI Mater. J. 97, 127-135 (2000).

${ }^{6}$ W.-J. Song, J. S. Popovics, J. C. Aldrin, and S. P. Shah, "Measurement of surface wave transmission coefficient across surface-breaking cracks and notches in concrete," J. Acoust. Soc. Am. 113, 717-725 (2003).

${ }^{7}$ J. O. Owino and L. J. Jacobs, "Attenuation measurements in cementbased materials using laser ultrasonics," J. Eng. Mech. 125, 637-647 (1999).

${ }^{8}$ Y.-C. Jung, W.-B. Na, T. Kundu, and M. Ehsani, "Damage detection in concrete using Lamb waves," Proceedings of SPIE-The International Society for Optical Engineering 3995, pp. 448-458, 2000.

${ }^{9}$ P. Anugonda, J. S. Wiehn, and J. A. Turner, "Diffusion of ultrasound in concrete," Ultrasonics 39, 429-435 (2001).

${ }^{10}$ Y. Kane, Master's thesis, University of Nebraska-Lincoln, 2003. 